\title{
Editorial
}

\section{Implantable loop recorder: towards a gold standard for the diagnosis of syncope?}

Syncope is a transient symptom and not a disease. Typically, patients are asymptomatic at the time of evaluation and the opportunity to capture a spontaneous event during diagnostic testing is rare. As a result, diagnostic evaluation has focused on the detection of abnormalities that could plausibly cause loss of consciousness. This type of reasoning necessarily leads to uncertainty in establishing a cause. In other words, the causal relation between an abnormality found during the diagnostic workup and syncope is often presumptive. Indeed, in the tests used to evaluate the aetiology of syncope, it is not possible to measure test sensitivity and specificity, owing to the lack of a reference standard for most of the tests. Because of the episodic behaviour of syncope, the opportunity of correlating the spontaneous syncopal episodes with an abnormal finding can be considered as a reference standard.

\section{Role of the implantable loop recorder}

An implantable ECG event monitor has recently become available (Reveal, Medtronic). This device is placed subcutaneously under local anaesthesia, and has a battery life of 15-18 months. The device has a solid state loop memory and, with the current version, the ECG of up to 40 minutes before and two minutes after activation can be stored. With these characteristics, if patients activate the device when consciousness has been restored, there is a high probability of having a correlation of ECG signals and syncope. In the first reported experience, the device was used in a heterogeneous population of 85 patients affected by unexplained syncope, ${ }^{1}$ which included patients with and without structural heart disease as well as patients with and without abnormalities in baseline ECG. Syncope-ECG correlation was achieved in $27 \%$ of patients and presyncope-ECG correlation in $32 \%$; the rhythm recorded during the event was heterogeneous, thus reflecting the various clinical settings of the population enrolled: 29 patients were in sinus rhythm, three had supraventricular tachycardia, and 18 had some type of "bradyarrhythmia", the origin of which was considered to be neurally mediated in seven patients. This study showed that patient activated, long term monitoring is feasible, helpful, safe, and probably cost effective.

From that initial experience it appeared that the implantable loop recorder might become the reference standard to be adopted when an arrhythmic cause of syncope is suspected but not sufficiently proven. There are several areas of interest:

- Tilt induced vasovagal syncope - We do not know whether the type of response induced during tilt testing (vasodepressor, mixed, cardioinhibitory) correlates with the spontaneous vasovagal attack. Knowing the exact mechanism of the spontaneous attack is of course of great practical importance in choosing the best treatment. One recent trial ${ }^{2}$ has shown that pacemaker therapy is highly efficacious in preventing syncopal recurrences in patients with cardioinhibitory vasovagal syncope. More generally, the same reasoning applies to all patients who have a likely diagnosis of neurally mediated syncope based on typical history (vasovagal syncope, situational syncope-that is, syncope that occurs during micturition, defaecation, swallowing or coughing - and carotid sinus syncope).

- Isolated syncope-This term can be applied to patients without apparent structural heart disease, normal ECG, and with a complete negative diagnostic work up, including tilt testing. In these patients there is a large consensus that the likely cause is neurally mediated.

- Bundle branch block, negative electrophysiologic study-In these patients, progression to complete atrioventricular (AV) block occurs in about $20 \%$ of cases within a few years, despite a complete negative electrophysiologic evaluation. $^{34}$

- Severe left ventricular dysfunction, non-sustained ventricular tachyarrhythmias, and negative electrophysiologic study - We know, for example, from the MUSTT (multicentre unsustained tachycardia trial) registry, ${ }^{5}$ that these patients have a lower risk of sudden death or arrhythmic events than those with a positive electrophysiologic study, but their risk still remains unacceptably high, being $24 \%$ and $32 \%$ at five years, respectively.

- Adenosine sensitive syncope-Some patients with unexplained syncope show an increased susceptibility to intravenous injection of adenosine or adenosine triphosphate (ATP), which results in a prolonged asystolic pause caused by paroxysmal AV block. ${ }^{67}$ The logical inference is that ATP testing can identify patients with syncope caused by transient AV block even when the electrophysiologic findings and other conventional tests are unremarkable. However, this remains a hypothesis to be confirmed by prospective studies aimed at demonstrating a similar abnormality during the spontaneous syncope.

The early lessons we are learning: the implantable loop recorder in "isolated" syncope and tilt positive syncope

ISSUE (international study on syncope of uncertain etiology) is an ongoing prospective study which aims to analyse the diagnostic yield of the implantable loop recorder in specific subgroups of patients with syncope of uncertain aetiology; the groups were predefined and the patients assigned to their groups at the time of enrolment. ${ }^{8}$ Overall, more than 200 patients have been enrolled and are being followed up. To date, we have only preliminary results on the subgroup of patients with isolated syncope (that is, no heart disease and complete negative work up including tilt testing) and on the subgroup of patients with positive response to tilt testing and no heart disease. The main findings are the following:

- In both groups, about two thirds of patients had no recurrence during follow up; of those who had recurrences, no patients suffered trauma or consequences caused by syncope. The low recurrence rate and the low risk of related injury we observed in the "real 


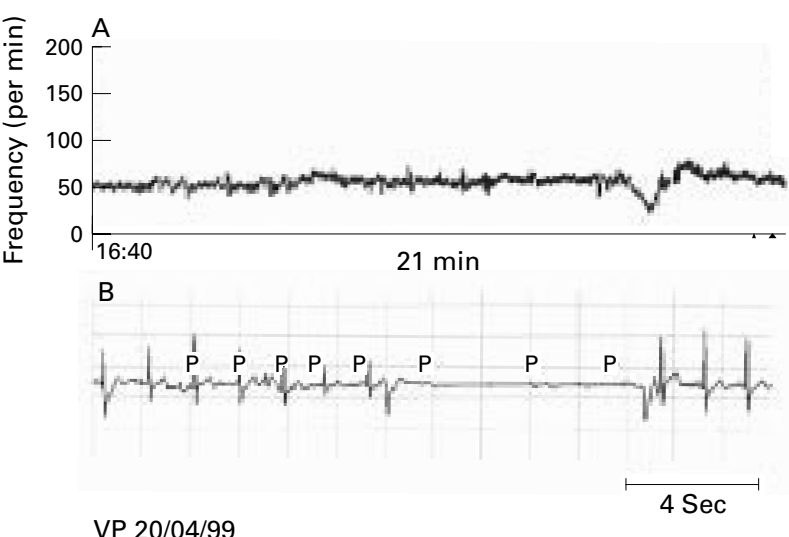

Figure 1 The most frequent syncopal pattern observed in patients with isolated syncope. (A) Heart rate trend during the whole 21 minute loop recording. Initially, the heart rate is stable at approximately 50 beats/min; before syncope, there is a progressively severe bradycardia lasting one minute followed by an asystolic episode that coincides with syncope. (B) The expanded ECG at the time of syncope shows an 8.5 second asystolic pause caused by AV block which coincides with a pronounced sinus rate slowing. The association, at the same time, of sinus bradycardia and AV block strongly suggests that a vagal reflex is the mechanism responsible for the event.

world" suggest that treatment, in particular pacemaker therapy, could be restricted only to those patients who have relapses, after diagnostic evaluation, or are at very high risk of injury.

- Among the patients with isolated syncope who had syncopal recurrences, three quarters had progressive sinus bradycardia more often followed by asystole, or progressive tachycardia followed by progressive bradycardia and more often asystole (with or without concomitant blocked $\mathrm{P}$ waves) (fig 1 ). These findings strongly suggest that the likely aetiology of syncope is neurally mediated and the most frequent mechanism is a dominant cardioinhibitory reflex with prolonged asystolic pauses.

- In the tilt positive patients, the results were very similar to those of the isolated syncope group, suggesting that the two groups have similar aetiologies (fig 2). Furthermore, in this group, an asystolic syncope was also recorded when the type of response to tilt testing was vasodepressor or mixed. Thus, it seems that the spontaneous syncope is much more frequently asystolic than one would expect on the basis of the results of tilt testing, which cannot be used to predict the type of response of

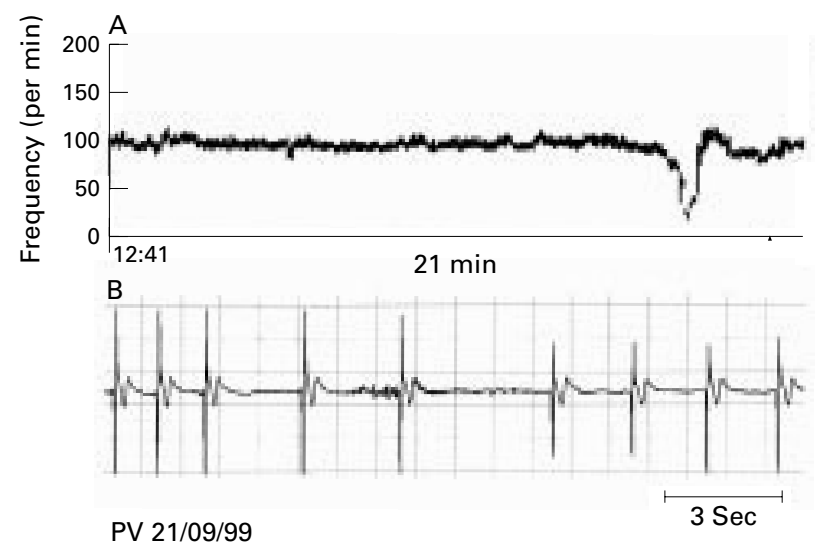

Figure 2 The most frequent syncopal pattern observed in the patients with tilt positive syncope. This particular patient had a

bradycardia-hypotension response during tilt testing. (A) Heart rate trend during the whole 21 minute loop recording. Initially, the heart rate is stable at approximately 100 beats/min; before syncope, there is a progressively severe bradycardia lasting two minutes. (B) The expanded ECG at the time of syncope shows a progressive sinus bradycardia with a maximum sinus pause of 4 seconds. the spontaneous attack. This finding explains why pacemaker therapy is more efficacious in preventing syncopal recurrences than expected. ${ }^{2}$

- In both groups, apart from the pattern described above, a normal sinus rhythm was frequently recorded at the time of syncope. Though not diagnostic, this finding allows us definitely to exclude an arrhythmia as the cause of syncope.

- One third of the patients had presyncope-ECG correlation. The recorded events were heterogeneous, normal sinus rhythm being the most frequent finding; paroxysmal tachyarrhythmias always caused presyncope. When syncope and presyncope were recorded in the same subject, different findings were frequently observed. Similar results had previously been obtained. ${ }^{2}$ Thus, presyncope was much less likely to be associated with arrhythmia than syncope was, and did not prove to be an accurate surrogate for syncope in establishing a diagnosis.

\section{Concerns about the use of the implantable loop recorder}

Apart from research purposes, ultimately the implantable loop recorder is used to find the most appropriate treatment of arrhythmias which cause syncope. Therefore, its use should be limited to patients with a high probability of arrhythmic syncope in whom the severity, frequency or hazardous nature of the episodes warrants specific treatment.

A diagnosis should be considered established only when syncopal episodes can be detected; in this case, the test can be regarded as the "gold standard". The recording of presyncope or asymptomatic arrhythmia - which is feasible in the new automatic version of the device-leaves the diagnosis uncertain.

Although the documentation of bradyarrhythmia concurrent with a syncopal episode is considered diagnostic, further evaluations may nevertheless be necessary in order to discriminate between an intrinsic cardiogenic abnormality and a neurogenic mechanism. Moreover, in the case of a patient with the recording of a cardioinhibitory neurally mediated syncope, one cannot exclude the possibility that the patient may also have different episodes - that is, vasodepressor.

Disadvantages of the implantable loop recorder include the need to implant an instrument in the patient, the lack of recording of any other concurrent physiological parameter (for example, blood pressure), and the high cost of the implantable device.

MICHELE BRIGNOLE

Department of Cardiology and Arrhythmologic Centre,

Ospedali Riuniti,

Lavagna, Italy

Department of Cardiology,

CARLO MENOZZI

Unit of Interventional Cardiology,

Ospedale S Maria Nuova,

Reggio Emilia, Italy

Unitat d'Aritmies,

ANGEL MOYA

Department of Cardiology,

Hospital General Vall d'Hebron,

Barcelona, Spain

Servicio de Cardiologia,

ROBERTO GARCIA-CIVERA

Hospital Clinico,

Valencia, Spain

On the behalf of the International Study on Syncope of Uncertain Etiology (ISSUE) Investigators 
1 Krahn AD, Klein GJ, Yee R, et al. Use of an extended monitoring strategy in patients with problematic syncope. Reveal investigators. Circulation

2 Sutton R, Brignole M, Menozzi C, et al. Dual-chamber pacing is efficacious in treatment of neurally-mediated tilt-positive cardioinhibitory syncope. Pacemaker versus no therapy: a multicentre randomized study. Circulation 2000;102:294-9.

3 Link M, Kim KM, Homoud M, et al. Long-term outcome of patients with syncope associated with coronary artery disease and a non-diagnostic electrophysiological evaluation. Am f Cardiol 1999;83:1334-7.

4 Gaggioli $\mathrm{G}$, Bottoni N, Brignole $\mathrm{M}$, et al. Progression to second- or third-degree atrioventricular block in patients electrostimulated for bundle branch block: a long-term study. G Ital Cardiol 1994:24:409-16.
5 Buxton A, Lee K, DiCarlo L, et al. Electrophysiologic testing to identify patients with coronary artery disease who are at risk for sudden death. $N$ Engl f Med 2000;342:1937-45.

6 Flammang D, Church T, Waynberger M, et al. Can adenosine 5'triphosphate be used to select treatment in severe vasovagal syndrome ? Circulation 1997;96:1201-8.

7 Brignole M, Gaggioli G, Menozzi C, et al. Adenosine-induced atrioventricular block in patients with unexplained syncope. The diagnostic value of ATP test. Circulation 1997;96:3921-7.

8 Moya A, Brignole M, Menozzi C, et al. Diagnostic yield of insertable loop recorder in patients with recurrent syncope: International study on syncope of uncertain etiology [abstract]. Eur Heart f 1999;20:442.

\section{Improvement in the pulmonary circulation following pulmonary thromboendarterectomy}

Chronic pulmonary thromboembolic disease is an insidious, life threatening condition that develops as a long term complication of the incomplete resolution of pulmonary embolism. Patients present with progressive breathlessness on exertion and the prognosis is poor owing to the development of pulmonary hypertension, with less than $30 \%$ five year survival. Pulmonary thromboendarterectomy has emerged as an effective treatment in selected patients.

Our patient was a 64 year old man with a five year history of progressive dyspnoea on effort following documented pulmonary embolism. He presented with severe exercise limitation (New York Heart Association functional class III) and signs of right ventricular hypertrophy. Invasive studies revealed a mean pulmonary arterial pressure of $65 \mathrm{~mm} \mathrm{Hg}$ and a cardiac index of $2.061 / \mathrm{min} / \mathrm{m}^{2}$. Contrast enhanced three dimensional magnetic resonance angiography (MRA) (below left) confirmed pulmonary

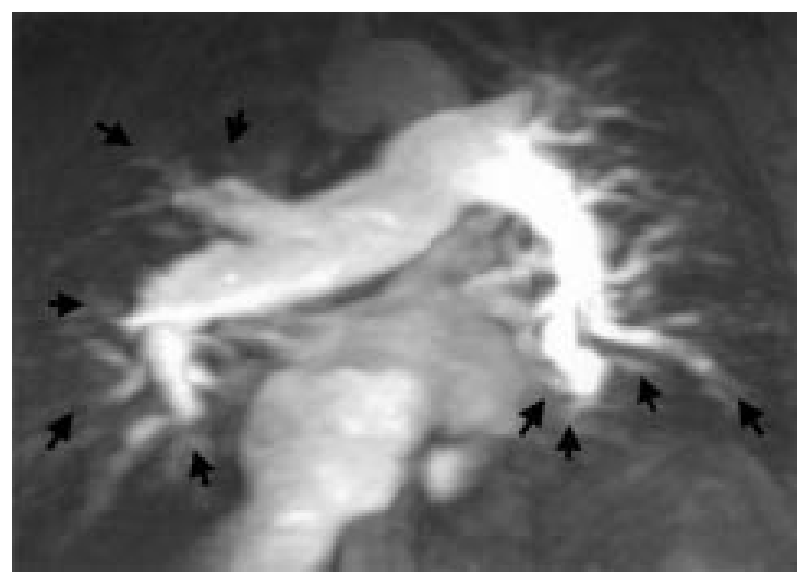

thromboembolic disease with multiple abrupt occlusions of segmental pulmonary arterial branches in both lower lobes and in the right upper lobe (arrows), proximal arterial dilatation, and vessel tortuosity. Pulmonary thromboendarterectomy was carried out under hypothermic circulatory arrest and an extensive cast of organised clot and thrombus was removed from the pulmonary arterial tree. The patient has made an uneventful recovery and at three months after surgery is asymptomatic, walking up to three miles daily. Mean pulmonary arterial pressure has fallen to $22 \mathrm{~mm} \mathrm{Hg}$ and the cardiac index has improved $(2.92$ $1 / \mathrm{min} / \mathrm{m}^{2}$ ). Repeat MRA demonstrates dramatic improvement in the pulmonary circulation with enhanced blood flow detected in subsegmental pulmonary arterial branches and in the pulmonary veins (below right).

RANA SAYEED RICHARD COULDEN JOHN DUNNING

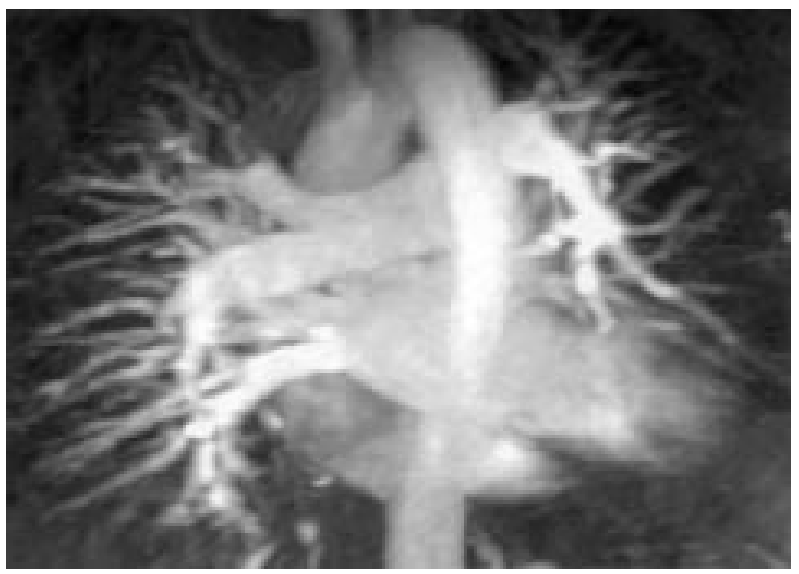

